\title{
Microencapsulation of Algal Oil Using Spray Drying Technology
}

\section{Wenyao Shao*, Xueshan Pan, Xiaoting Liu, Feixue Teng and Shuai Yuan}

Department of Chemical and Biochemical Engineering, College of Chemistry and Chemical Engineering, Xiamen University, Xiamen, 361005 Fujian, PR China

Received: July 30, 2017 Accepted: January 25, 2018
*Corresponding author:

Phone: +86 5922188848 ;

Fax: +86 5922188848 ;

E-mail:wyshao@xmu.edu.cn

ORCID IDs: 0000-0001-8308-8340 (Shao), 0000-0002-9810-3475 (Pan), 0000-0001-9352-4804 (Liu), 0000-0001-7225-9829 (Teng), 0000-0002-2273-5249 (Yuan)

Paper was presented at the 7th International Forum on Industrial Bioprocessing - IFIBiop 2017, May 21-24, 2017, Wuxi, PR China

\section{SUMMARY}

This work aims at developing a process of microencapsulation of algal oil containing $\geq 40 \%$ docosahexaenoic acid (DHA) using spray drying technology. Purity Gum ${ }^{\circledast} 2000$ and Capsul $^{\circledR}$, both obtained from waxy corn starch, were chosen as the encapsulation materials. The effects of emulsification conditions on the droplet size, stability, viscosity and surface tension, and the effects of spraying conditions on the particle size, moisture content and surface oil content were investigated successively. The morphology of emulsion droplets and the microcapsules was observed by optical microscope and scanning electron microscopy. The results showed that the produced spherical microcapsules were smooth and free of pores, cracks, and surface indentation when shear velocity was $8.63 \mathrm{~m} / \mathrm{s}$ in the first step of emulsification, homogenization pressure was $1.75 \cdot 10^{8} \mathrm{~Pa}$ and number of passes through homogenization unit was six for fine emulsification, rotational speed of spray disk was $400 \mathrm{~s}^{-1}$, and air inlet temperature was $170^{\circ} \mathrm{C}$. Therefore, it was concluded that the emulsification and encapsulation of algal oil containing DHA with above process was feasible.

Key words: microencapsulation, docosahexaenoic acid (DHA), algal oil, spray drying, emulsification, morphology of DHA microcapsule

\section{INTRODUCTION}

Docosahexaenoic acid (DHA, 22:6 n-3) is an important polyunsaturated fatty acid for human body, which is essential for the development of eye, neural system, and mammalian nervous system, and can lower the incidence of arthritis, hypertension, thrombosis and arteriosclerosis (1,2), as well as cardiovascular diseases (3), inflammation (4) and cancer. As the main type of long-chain polyunsaturated fatty acids (PUFAs) in the brain, DHA accounts for about $15 \%$ of total fatty acids in the brain. Nowadays, PUFA-producing microorganisms are being investigated because they are potential alternative sources of PUFAs. This is because fish oil, a traditional source of PUFAs, is considered as a depleted resource due to the high demand of PUFAs, and its composition and quality also vary a lot (5). However, unsaturated bonds in molecular structure make DHA vulnerable to oxidizing agents, light and high temperature, which leads to the formation of toxic hydroperoxides, foul taste, and limited shelf life of the products (6). Therefore, DHA needs to be protected from oxidation in some form before industrial application.

In the field of food processing, microencapsulation technology is extensively involved in preventing food spoilage, volatile loss and loss of properties of essential oils (7). In microencapsulation, sensitive ingredients or core materials entrapped in a protective polymer encapsulating agent or wall material can be protected from degenerative reactions and unfavourable environmental conditions, so that their stability and application range are enhanced (8). Therefore, all kinds of microencapsulation technologies have been developed, such as solvent dispersion/evaporation, phase separation (coacervation), co-crystallization, interfacial polymerization, spray-drying, etc., among which spray-drying is most generally applied in the food industry, because of the possibility of continuous production and easy industrialization $(9,10)$. Normally, an initial emulsification step is included in spray drying, when the wall 
materials are used as a stabilizer for core materials. Then, emulsions are converted into a free-flowing powder. Therefore, in order to acquire desired powders by further spray drying, emulsification step and the emulsion properties are critical, such as its stability, size and viscosity. Some carbohydrates such as starches have been widely used as encapsulating agents (11), because they have many of the needed properties, such as low viscosity at high solid content and high solubility. In this study, Purity Gum ${ }^{\circledR} 2000$ and Capsul ${ }^{\circledR}$ obtained from waxy corn starch were used as encapsulation materials, and the emulsification and encapsulation process of DHA were investigated to obtain optimal process conditions.

\section{MATERIALS AND METHODS}

\section{Materials}

Purity Gum ${ }^{\circledR} 2000$ and Capsul ${ }^{\circledR}$ were purchased from Ingredion Inc., Westchester, IL, USA. Algal oil containing $\geq 40 \%$ docosahexaenoic acid (DHA) was supplied by Xiamen Kingdomway Group Company, Xiamen, PR China. All materials were of food grade.

\section{Emulsion preparation}

Purity Gum ${ }^{\circledR} 2000(9 \mathrm{~g})$ and Capsul ${ }^{\circledR}(13.5 \mathrm{~g})$ were each dissolved in $127.5 \mathrm{~mL}$ of hot deionized water $\left(75^{\circ} \mathrm{C}\right)$ and stirred for $15 \mathrm{~min}$ to form an aqueous solution. Then, $10 \mathrm{~g}$ of algal oil were added dropwise to the solution, and the mixtures were homogenized to form the oil-in-water emulsions. There are two steps for the emulsion preparation: first, the emulsions were homogenized using an Ultra Turrax T25 (IKA Co., Staufen, Germany) homogenizer for $5 \mathrm{~min}$. The effects of rotational speed of high-shear emulsifier (IKA Co.) on the emulsions were investigated; second, fine emulsions were formed after the coarse emulsions were passed through a high-pressure microfluidizer (M-110P Basic; Microfluidics ${ }^{\mathrm{TM}}$ International Corp., Newton, MA, USA) at different number of times and at various homogenization pressures. The effects of pressure and number of passes on the emulsions were explored in this study.

The viscosity of the emulsions was measured with a digital viscometer (Shanghai FANGRUI Instrument Co. Ltd, Shanghai, PR China). Surface tension of the emulsions was measured by maximum bubble pressure method at $20^{\circ} \mathrm{C}$. The size distribution of the emulsion droplets was measured by dynamic light scattering technique (zeta potential analyzer Zeta PALS; Brookhaven Instruments Corporation, Holtsville, NY, USA) at a fixed angle of $90^{\circ}$ at $25^{\circ} \mathrm{C}$. The morphology of the emulsion droplets was observed using an optical microscope (BK-POLR; Chongqing Optec Instrument Co., Ltd, Chongqing, PR China), and photographed using digital camera (TYP112; Leica, Tokyo, Japan).

The centrifugal separation method was used to assess the emulsion stability (12). A mass of $0.90 \mathrm{~g}$ of primary emulsions was transferred to graduated centrifuge tubes which were placed in aqueous bath at $60^{\circ} \mathrm{C}$ for $5 \mathrm{~min}$, and then centrifuged (5810R; Eppendorf, Hamburg, Germany) for $10 \mathrm{~min}$ at 4000×g. The volumes of separated phases were recorded, and the emulsion stability was calculated as the percentage of the total water still emulsified after treatment using the following equation:

$$
\text { Emulsion stability }=\left(1-\frac{V_{1}}{V_{0}}\right) \cdot 100
$$

where $V_{1}$ is the volume of emulsions resolved after centrifugation, and $V_{0}$ is the initial emulsion volume.

\section{Microcapsule preparation}

To produce microcapsules containing DHA, the emulsions were spray-dried by co-current drying using a spray dryer (GZ-5; Wuxi Sun Dryness Facility Factory, Wuxi, PR China). First, blown by the inlet fan, the ambient air was heated by a resistor in the air heating chamber. Second, the heated air was blown into the top of the drying chamber, where the emulsions were sprayed into the air stream. Then, with a hygienic progressing cavity pump (KB-SL; Pumpenfabrik Wangen $\mathrm{GmbH}$, Wangen, Germany), the emulsions were atomized and sprayed into the drying chamber, where droplets were dried and yielded dried powder and dust. After that, the powder, dust and air were pulled to the bottom of drying chamber and then to the cyclone separator by the exhaust fan. In the cyclone separator the powder and dust were separated. The powder was collected in the cyclone collector, and the exhaust air was expelled through a filter bag to the atmosphere. The effects of rotational speed of spray disk and air inlet temperature on the microcapsule were investigated in this study.

\section{Microcapsule characterization}

The particle size distribution was measured by high resolution laser particle size analyzer (Saturn DigiSizer 5200; Micromeritics Instrument Corp., Norcross, GA, USA). The particle size was calculated as the mean volumetric size $d_{43}$ (De Brouckere mean diameter). The morphology of the spray-dried microcapsules was observed by scanning electron microscopy (SEM, S-4800; Hitachi, Tokyo, Japan) at 15 kV. After sputtering with gold, the microcapsule surfaces were observed and photographed using a digital camera (TYP112; Leica).

\section{Encapsulation efficiency and moisture content}

With a slight modification of the description by Bae and Lee (13), microencapsulation efficiency (ME) was calculated by measuring the mass of surface and total oil, according to the following equation:

$$
\mathrm{ME}=\frac{m_{\mathrm{t}}-m_{\mathrm{s}}}{m_{\mathrm{t}}} \cdot 100
$$

where $m_{t}$ and $m_{s}$ are the total oil and surface oil mass, respectively.

After stirring $3 \mathrm{~g}$ of microcapsule with $20 \mathrm{~mL}$ of hexane in a covered $100-\mathrm{mL}$ beaker at $25^{\circ} \mathrm{C}$ for $10 \mathrm{~min}$, the suspension 
was filtered and the residue was rinsed thrice, each time with $20 \mathrm{~mL}$ of hexane. After the residual air-powder was dried for $30 \mathrm{~min}$, the filtrate solution containing the extracted oil was transferred to a clean beaker to evaporate, which was then dried to constant mass at $105^{\circ} \mathrm{C}$. The surface oil mass was determined according to the difference between the initial clean beaker mass and that containing the extracted oil residue (14).

The moisture content of the microcapsules was obtained gravimetrically. About $5 \mathrm{~g}$ of dry sample were placed into an air oven at $105^{\circ} \mathrm{C}$ for $3 \mathrm{~h}$. Formula for calculating the moisture content was as follows:

$$
w(\text { moisture })=\left(\frac{m_{0}-m_{1}}{m_{0}}\right) \cdot 100
$$

where $m_{0}$ and $m_{1}$ are the mass of sample before and after the treatment, respectively.

\section{RESULTS AND DISCUSSION}

\section{Emulsion preparation}

The parameters of emulsification, such as shear velocity, homogenization pressure and number of passes through the homogenization unit, in the preparation of microcapsules can influence further steps of preparation of encapsulated oil containing $\geq 40 \% \mathrm{DHA}$ as a health care product $(15,16)$. Therefore, in this study, the optimal operating conditions were investigated.

Fig. 1 shows the effects of shear velocity on the droplet size and stability of the emulsions. Results showed that the droplet size decreased from 16.86 to $8.70 \mu \mathrm{m}$ when shear velocity increased from 4.65 to $9.96 \mathrm{~m} / \mathrm{s}$, which is consistent with the fact that the emulsion droplet size can be reduced by increasing the amount of energy supplied during emulsification (17). On the other hand, the shear velocity had no significant impact on the stability of the emulsions. The corresponding optical micrographs obtained at various shear velocities are shown in Figs. 2a-e. Comparing Figs. $2 \mathrm{~d}$ and $2 \mathrm{e}$, it can be seen that the

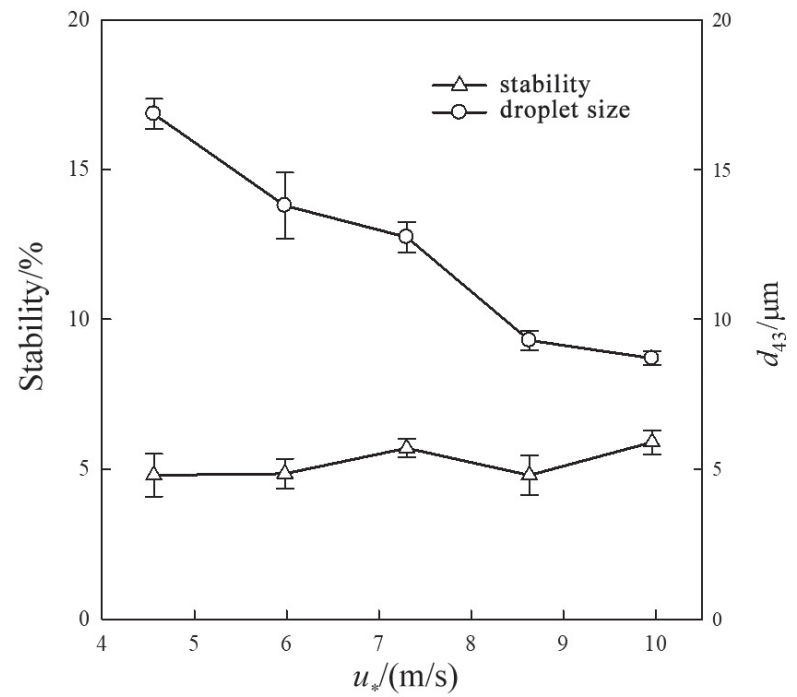

Fig. 1. Effect of the shear velocity $\left(u_{*}\right)$ on the stability and droplet size $\left(d_{43}\right)$ of the emulsions reduction in droplet size was not significant when the shear velocity was increased from 8.63 to $9.96 \mathrm{~m} / \mathrm{s}$. Therefore, $8.63 \mathrm{~m} / \mathrm{s}$ was chosen as shear velocity for the first step of emulsification. To obtain uniform size emulsions, a subsequent secondary step, a microfluidic emulsification, was carried out.
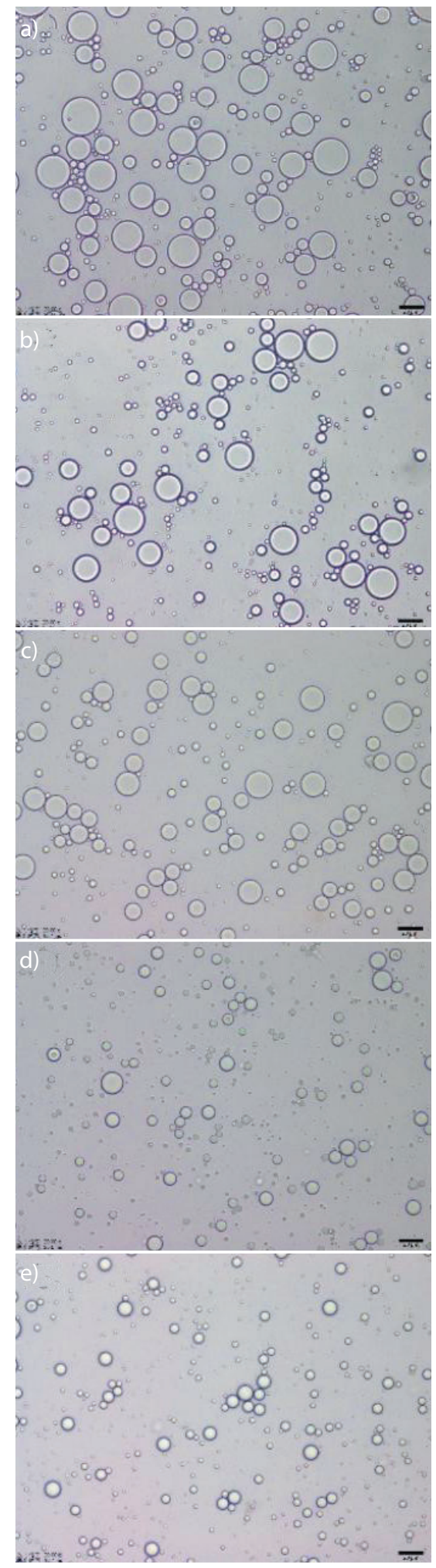

Fig. 2. Micrographs of the emulsions obtained at various shear velocities: a) 4.65 , b) 5.98 , c) 7.3 , d) 8.63 and e) $9.96 \mathrm{~m} / \mathrm{s}$. Scale bar is $20 \mu \mathrm{m}$ 
Fig. 3 and Fig. 4 show the effects of homogenization pressure (number of passes through homogenization unit was set at 1) on the mean droplet size and stability, as well as on the viscosity and surface tension of the emulsions obtained after secondary step of emulsification, respectively. Compared to the coarse emulsions obtained by using a shear emulsifier, a significant decrease in droplet size from $9.5 \mu \mathrm{m}$ to less than $100 \mathrm{~nm}$, and a significant increase in stability from 5 to more than $60 \%$ were observed after microfluidic emulsification. Fig. 3 also shows that the droplet size decreased with the increase of homogenization pressure, which indicated that the microfluidizer could form small droplet sizes in emulsions efficiently. The increase in the magnitude of the disruptive forces generated within the homogenization chamber results in

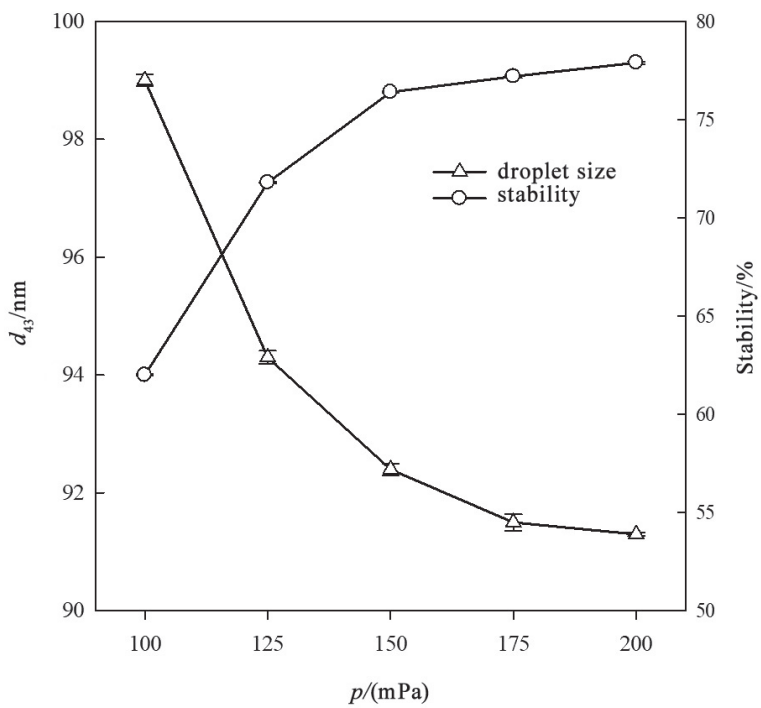

Fig. 3. Effect of homogenization pressure on the droplet size $\left(d_{43}\right)$ and stability of the emulsions

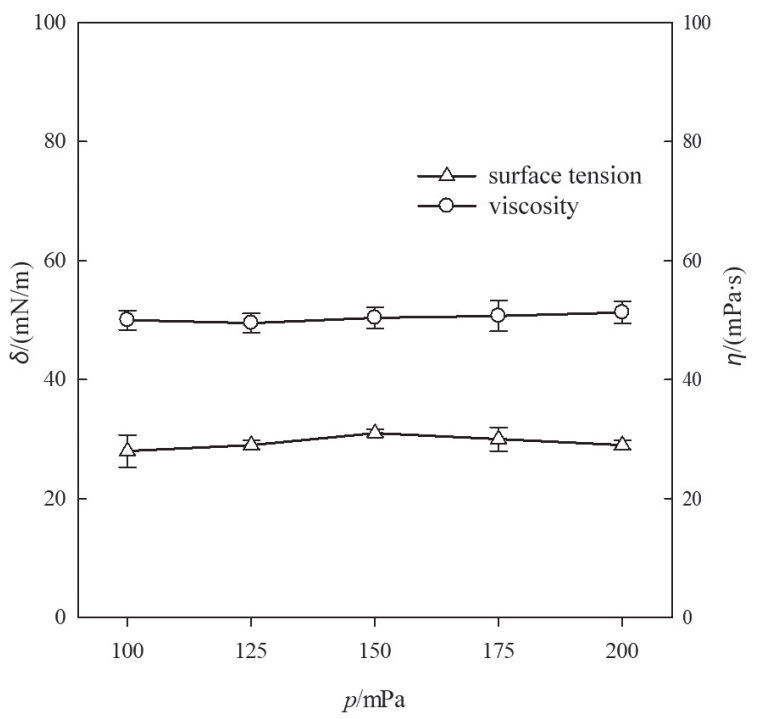

Fig. 4. Effect of homogenization pressure on the viscosity and surface tension of the emulsions the decrease of droplet size with increasing pressure. However, the droplet size and stability of the emulsions did not change too much when the pressure increased from $1.75 \cdot 10^{8}$ to $2 \cdot 10^{8} \mathrm{~Pa}$. This may be due to the fact that under a specific set of emulsification conditions, there is a minimum limit in droplet size, and thus it would be inefficient to emulsify the system, or it may result in bigger size because of poor stabilization of the newly formed droplets (18). The pressure had no significant effect on the viscosity and surface tension of the emulsions, as shown in Fig. 4. Therefore, homogenization pressure of $1.75 \cdot 10^{8} \mathrm{~Pa}$ was selected for the remainder of the experiments.

To further optimize the fine emulsification conditions, the effect of the number of passes through homogenization unit on the emulsions was investigated (Fig. 5 and Fig. 6.) It was observed that the droplet size decreased from around 92 to $72 \mathrm{~nm}$ with the increase of the number of passes from 1 to 6 (Fig. 5), which is in agreement with previous studies $(18,19)$. However, the droplet size increased after 6 passes. As far as we know, the emulsification process includes two steps: (i) droplets are deformed and disrupted, increasing the specific surface area of the emulsions, and (ii) the newly formed interface is stabilized by an emulsifier to prevent re-coalescence of the fresh droplets. When the timescale of surfactant absorption is longer than that of collision, the fresh interface will not be completely covered, which would lead to re-coalescence. Therefore, the 'over-processing' phenomenon may occur, which indicates that the obtained emulsions have bigger droplet size than expected, even with the increased energy input (20). It was also found that the greatest stability of the emulsions was obtained at six passes compared to other numbers of passes, and the number of passes had no apparent impact on the viscosity and surface tension of the emulsions (Fig. 6). Therefore, six passes through homogenization unit was chosen as the optimum for the homogenization.

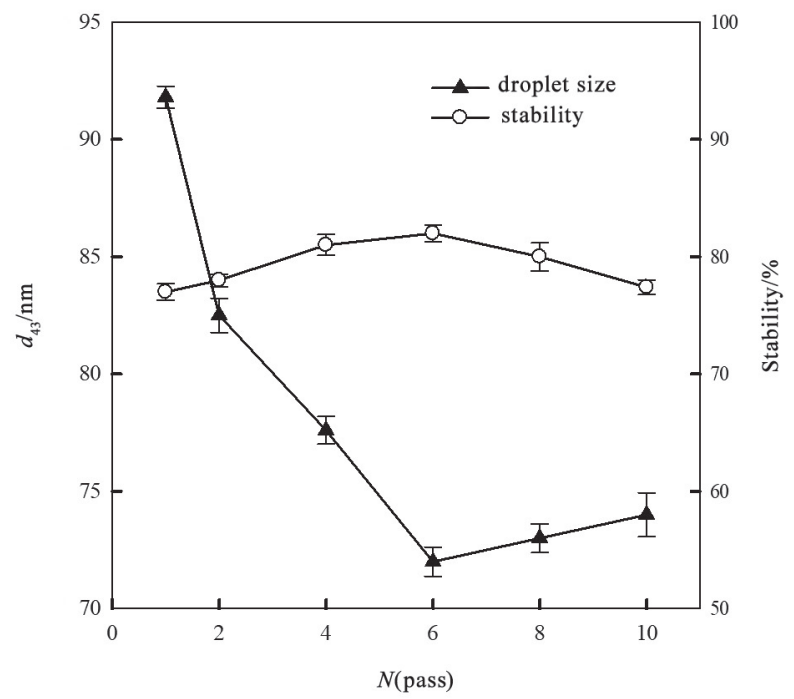

Fig. 5. Effect of the number of passes through the homogenization unit on droplet size $\left(d_{43}\right)$ and stability of the emulsions 


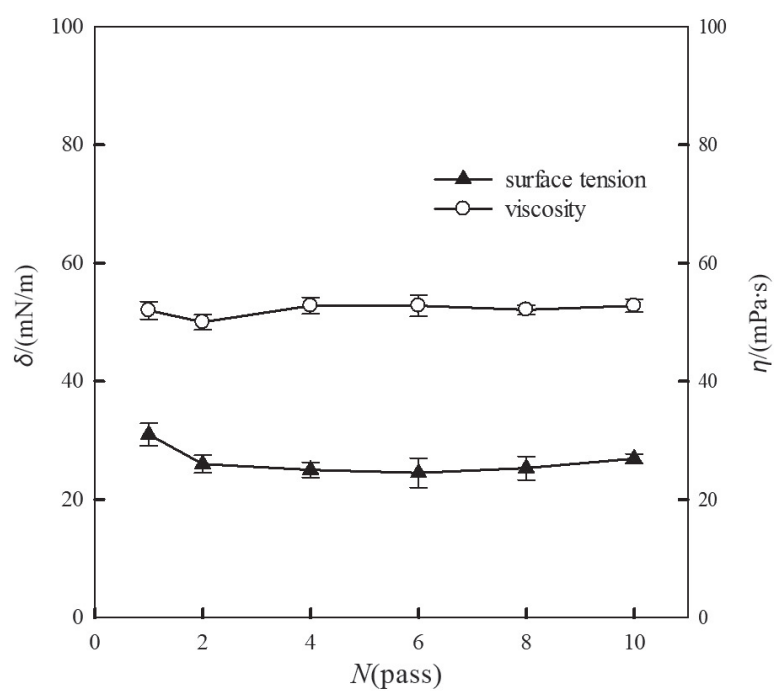

Fig. 6. Effect of the number of passes through the homogenization unit on the viscosity and surface tension of the emulsions

\section{Microcapsule preparation}

After the preparation of the emulsions by shear emulsifier and microfluidic emulsification, microencapsulation of algal oil containing $\geq 40 \%$ DHA was done by spray drying. To optimize the spray drying conditions, experiments were carried out to explore the effects of rotational speed of spray disk and air inlet temperature on the properties of produced microcapsules, including particle size, moisture content and surface oil content.

Fig. 7 shows the effect of rotational speed of spray disk on the microcapsule properties when the air inlet temperature and emulsion feeding rate were $160{ }^{\circ} \mathrm{C}$ and $25 \mathrm{~g} / \mathrm{min}$, respectively. It was shown that with the increase of rotational speed, the size and moisture content of the microcapsule decreased, while the surface oil content increased. Small droplets are formed at high rotational speed, which results in powder. However, due to the decrease of particle size, the oil cannot be encapsulated completely, which results in larger

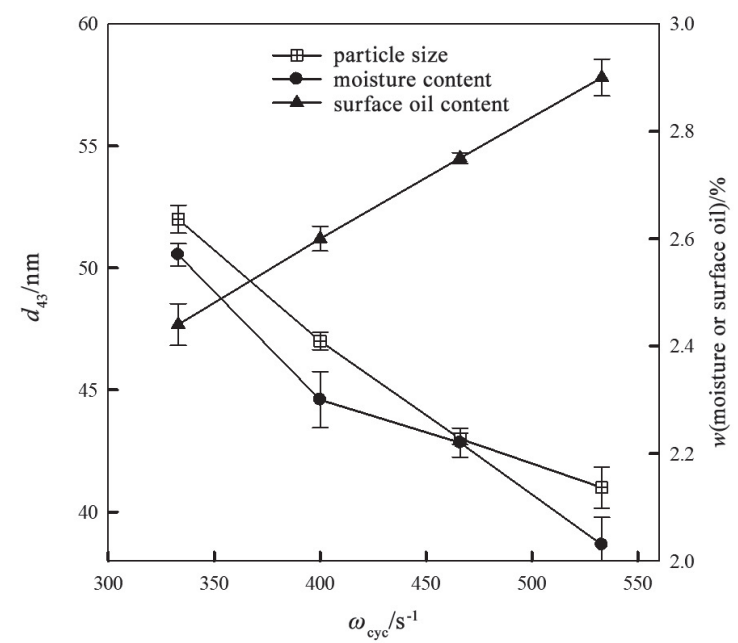

Fig. 7. Effect of rotational speed of spray disk ( $\left.\omega_{\text {cyc }}\right)$ on microcapsule properties surface oil content. Therefore, $400 \mathrm{~s}^{-1}$ was chosen as the optimal rotational speed for the encapsulation.

The air inlet temperature is another major factor in microencapsulation. Fig. 8 shows the effect of air inlet temperature on the microcapsule properties when the rotating speed and emulsion feeding rate were $400 \mathrm{~s}^{-1}$ and $25 \mathrm{~g} / \mathrm{min}$, respectively. It was found that with the increase of inlet temperature, the moisture content decreased while the surface oil mass fraction increased. However, the inlet temperature had no significant effect on the particle size. In fact, air inlet temperature is in direct proportion to the microcapsule drying rate and the final water content. With low air inlet temperature, microcapsules have high-density membranes, high water content, poor fluidity, and easy agglomeration because of the low evaporation rate. On the contrary, with high air inlet temperature, microcapsules have low-density membrane, inducing subsequent premature release and degradation of encapsulated ingredient or loss of volatiles because of the excessive evaporation. Based on the above experiment, 170 ${ }^{\circ} \mathrm{C}$ was chosen as the optimal inlet temperature.

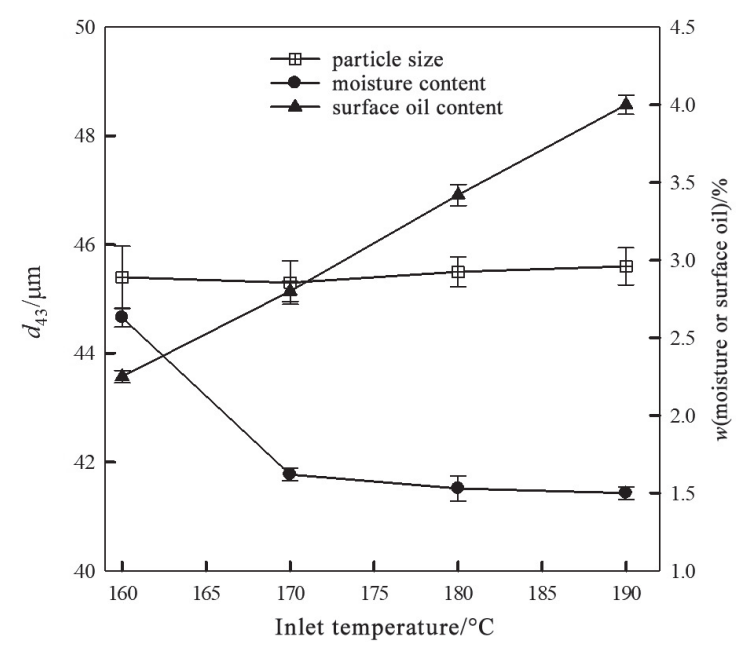

Fig. 8. Effect of air inlet temperature on the microcapsule properties

The surface morphology of microcapsules containing $\geq 40 \%$ DHA was verified by using scanning electron microscopy (SEM) (Fig. 9). It was concluded that the emulsification and encapsulation of oil containing DHA using the above process were feasible, and the produced spherical microcapsules were smooth and free of pores, cracks and surface indentation.

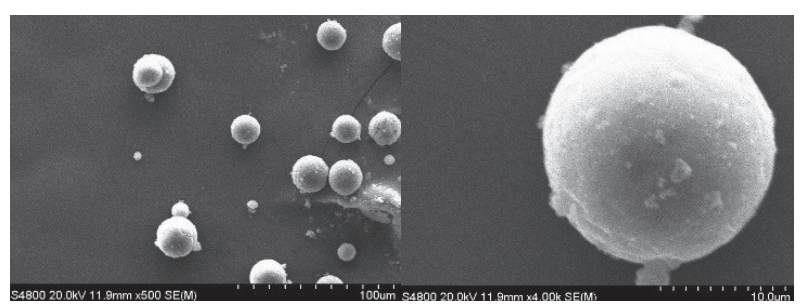

Fig. 9. Scanning electron microscopy (SEM) images of the algal oil containing $\geq 40 \%$ docosahexaenoic acid (DHA) microcapsules 


\section{CONCLUSIONS}

A process of microencapsulation of algal oil containing $\geq 40$ $\%$ docosahexaenoic acid (DHA) using spray drying was developed in this study. Purity Gum ${ }^{\circledR} 2000$ and Capsul ${ }^{\circledR}$ were chosen as the encapsulation materials. The optimal conditions were: shear velocity of $8.63 \mathrm{~m} / \mathrm{s}$ for the first step of emulsification, homogenization pressure of $1.75 \cdot 10^{8} \mathrm{~Pa}$ and number of passes through the homogenization unit were six for fine emulsification, rotational speed of spray disk $400 \mathrm{~s}^{-1}$, and air inlet temperature $170^{\circ} \mathrm{C}$. Under the optimal conditions, the produced microcapsules with spherical shape were smooth without pores, cracks or surface indentation, which indicated the emulsification and encapsulation of algal oil containing DHA with the above process was feasible.

\section{ACKNOWLEDGEMENTS}

The authors gratefully acknowledge the financial support by the Fundamental Research Funds for the Central Universities (No. 20720170027) and National Natural Science Foundation Youth Fund of China (No. 21406185).

\section{REFERENCES}

1. Arab-Tehrany $E$, Jacquot $M$, Gaiani C, Imran M, Desobry $S$, Linder M. Beneficial effects and oxidative stability of omega-3 long-chain polyunsaturated fatty acids. Trends Food Sci Technol. 2012;25:24-33.

https://doi.org/10.1016/j.tifs.2011.12.002

2. Sun L, Ren L, Zhuang X, Ji X, Yan J, Huang H. Differential effects of nutrient limitations on biochemical constituents and docosahexaenoic acid production of Schizochytrium sp. Bioresour Technol. 2014;159:199-206.

https://doi.org/10.1016/j.biortech.2014.02.106

3. Schram LB, Nielsen CJ, Porsgaard T, Nielsen NS, Holm R, Mu $H$. Food matrices affect the bioavailability of $(n-3)$ polyunsaturated fatty acids in a single meal study in humans. Food Res Int. 2007;40:1062-8.

https://doi.org/10.1016/j.foodres.2007.06.005

4. Mickleborough TD. Anti-inflammatory effects of polyunsaturated fatty acids on the inflammatory response in asthma. Agro Food Ind Hi Tec. 2009;20:10-2.

5. Sijtsma $L$, de Swaaf ME. Biotechnological production and applications of the $\omega-3$ polyunsaturated fatty acid docosahexaenoic acid. Appl Microbiol Biotechnol. 2004;64:146-53. https://doi.org/10.1007/s00253-003-1525-y

6. Kolanowski W, Berger S. Possibilities of fish oil application for food products enrichment with omega-3 PUFA. Int J Food Sci Nutr. 1999;50:39-49.

https://doi.org/10.1080/096374899101409

7. Bakry AM, Abbas S, Ali B, Majeed H, Abouelwafa MY, Mousa A, Liang L. Microencapsulation of oils: A comprehensive review of benefits, techniques, and applications. Compr Rev Food Sci F. 2016;15:143-82.

https://doi.org/10.1111/1541-4337.12179
8. Hogan SA, McNamee BF, O'Riordan ED, O'Sullivan M. Emulsification and microencapsulation properties of sodium caseinate/carbohydrate blends. Int Dairy J. 2001;11:137-44. https://doi.org/10.1016/S0958-6946(01)00091-7

9. Jimenez M, García HS, Beristain Cl. Spray-drying microencapsulation and oxidative stability of conjugated linoleic acid. Eur Food Res Technol. 2004;219:588-92. https://doi.org/10.1007/s00217-004-0992-4

10. Rodríguez-Huezo ME, Pedroza-Islas R, Prado-Barragán LA, Beristain $\mathrm{Cl}$, Vernon-Carter EJ. Microencapsulation by spray drying of multiple emulsions containing carotenoids. J Food Sci. 2004;69:351-9.

https://doi.org/10.1111/j.1365-2621.2004.tb13641.x

11. Wang R, Tian Z, Chen L. A novel process for microencapsulation of fish oil with barley protein. Food Res Int. 2011;44:2735-41.

https://doi.org/10.1016/j.foodres.2011.06.013

12. Spiecker PM, Gawrys KL, Trail CB, Kilpatrick PK. Effects of petroleum resins on asphaltene aggregation and water-in-oil emulsion formation. Colloids Surf A. 2003;220:9-27. https://doi.org/10.1016/S0927-7757(03)00079-7

13. Bae EK, Lee SJ. Microencapsulation of avocado oil by spray drying using whey protein and maltodextrin. J Microencapsul. 2008;25:549-60. https://doi.org/10.1080/02652040802075682

14. Jafari SM, Assadpoor E, Bhandari B, He Y. Nano-particle encapsulation of fish oil by spray drying. Food Res Int. 2008;41:172-83.

https://doi.org/10.1016/j.foodres.2007.11.002

15. Schultz S, Wagner G, Urban K, Ulrich J. High-pressure homogenization as a process for emulsion formation. Chem Eng Technol. 2004;27:361-8.

https://doi.org/10.1002/ceat.200406111

16. Urban K, Wagner G, Schaffner D, Roglin D, Ulrich J. Rotor-stator and disc systems for emulsification processes. Chem Eng Technol. 2006;29:24-31. https://doi.org/10.1002/ceat.200500304

17. Jafari SM, Assadpoor E, He Y, Bhandari B. Re-coalescence of emulsion droplets during high-energy emulsification. Food Hydrocoll. 2008;22:1191-202. https://doi.org/10.1016/j.foodhyd.2007.09.006

18. Qian C, McClements DJ. Formation of nanoemulsions stabilized by model food-grade emulsifiers using high-pressure homogenization: Factors affecting particle size. Food Hydrocoll. 2011;25:1000-8.

https://doi.org/10.1016/j.foodhyd.2010.09.017

19. Tan CP, Nakajima M. $\beta$-Carotene nanodispersions: Preparation, characterization and stability evaluation. Food Chem. 2005;92(4):661-71. https://doi.org/10.1016/j.foodchem.2004.08.044

20. Marie P, Perrier-Cornet JM, Gervais P. Influence of major parameters in emulsification mechanisms using a high-pressure jet. J Food Eng. 2002;53:43-51. https://doi.org/10.1016/S0260-8774(01)00138-8 